\title{
A Proposal for Optical Experiments on Nonlocality with a Linear Anisotropic Crystal
}

\author{
V.K.Ignatovich ${ }^{1 *}$ and F.V.Ignatovich ${ }^{2}$ \\ ${ }^{1}$ Joint institute for Nuclear Research, Dubna, Russia \\ ${ }^{2}$ Lumetrics inc, Rochester, N.Y., USA \\ Email: v.ignatovi@gmail.com
}

\begin{abstract}
Propagation of two beams through a birefringent calcite crystal is considered. Experiments are proposed the same as the ones performed with down conversion photons in nonlinear crystals.
\end{abstract}

Keywords: Optics, birefringence, interference, coincidence, entanglement.

\section{Introduction}

Electromagnetic waves and photons, they are an endless source of research of themselves and as a tool of investigation in many branches of physics. Here their own properties are under consideration, the properties, which are related to such fundamental topics as photon's pair correlations $[1,2,3,4,5]$, interference and coincidence counts. However, instead of using a nonlinear crystal $[1,2,3,4]$ we propose here to use a linear one. It became possible after development of the new theory [6] of optics in anisotropic media. The investigations can be performed along the same scheme as in [3], shown in Fig.1

It is possible to measure an interference in every detector in dependence of position of the beam splitter, the coincidence probability and to check the Bells inequalities. Below we present calculation of the exiting two beams angular separation after a uniaxial birefringent crystal with tensor $\varepsilon$ represented as

$$
\varepsilon_{i j}=\epsilon_{0} \delta_{i j}+\epsilon^{\prime} a_{i} a_{j}
$$

where $\epsilon_{0}$ is isotropic part, and anisotropy is characterized by the unit vector $\boldsymbol{a}$ with components $a_{i}$ and by anisotropy parameter $\epsilon^{\prime}$. We will consider calcite for which $\epsilon_{0}=2.751$ and $\eta=\epsilon^{\prime} / \epsilon_{0}=-0.197$.

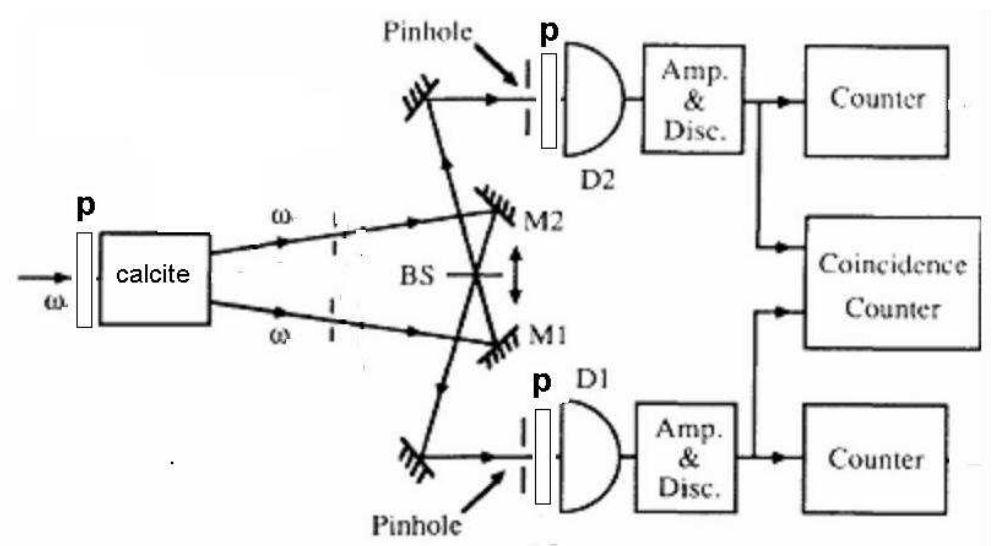

Figure 1. Outline of the experimental setup. It differs from that shown in [3] by absence of different filters. The two photons exiting from the crystal are related to birefringence and not to parametric down conversion. Polarizers $\mathbf{P}$ can change polarization. Variation of polarization of the incident beam changes the relative intensity of two beams at the calcite exit. 


\section{Splitting of Waves by an Anisotropic Crystal}

Let's consider a plane wave incident on the left side of the prism, as shown in Fig.2. Let the the anisotropy vector be parallel to the left surface of the prism and at $\pi / 4$ to the incidence plane i.e. $\boldsymbol{a} \cdot \boldsymbol{l}=\boldsymbol{a} \cdot \boldsymbol{t}=\sqrt{2} / 2$. Inside the prism two modes will appear: $\boldsymbol{E}_{1}$ with wave vector $\boldsymbol{k}_{1}$ and polarization

$$
e_{1}=[\boldsymbol{a} \times \boldsymbol{\kappa}],
$$

which is orthogonal to the plane, containing the anisotropy vector $\boldsymbol{a}$ and the wave vector $\boldsymbol{k}$. Here $\boldsymbol{\kappa}=\boldsymbol{k} / k$ is the unit vector in the direction of the wave propagation, $k=k_{0} \sqrt{\epsilon_{0}}$ and $k_{0}=\omega / c$. Another mode, $\boldsymbol{E}_{2}$, has polarization in the above plane along the vector

$$
\boldsymbol{e}_{2}=\boldsymbol{a}-\boldsymbol{\kappa}(\boldsymbol{\kappa} \cdot \boldsymbol{a}) \frac{1+\eta}{1+\eta(\boldsymbol{\kappa} \cdot \boldsymbol{a})^{2}} \equiv \boldsymbol{a}-\boldsymbol{\kappa}(\boldsymbol{\kappa} \cdot \boldsymbol{a}) \epsilon(\chi) / \epsilon_{0},
$$

where $\eta=\epsilon^{\prime} / \epsilon_{0}$ and $\chi$ is the angle between unit vectors $\boldsymbol{\kappa}$ and $\boldsymbol{a}$. This mode propagates with $k=k_{0} \sqrt{\epsilon(\chi)}$, where

$$
\epsilon(\chi)=\epsilon_{0} \frac{1+\eta}{1+\eta \cos ^{2} \chi}
$$

is the value of anisotropic dielectric permittivity. It is convenient to call the mode $\boldsymbol{e}_{1}$ as orthogonal, and the mode $\boldsymbol{e}_{2}$ as mixed because in general it has a longitudinal component along the wave vector $\boldsymbol{k}$. Such a nomenclature looks more appropriate than the ordinary and extraordinary commonly used.

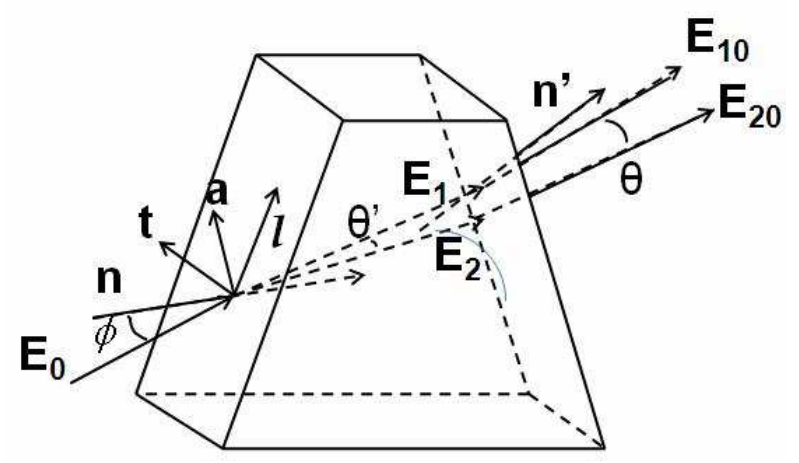

Figure 2. Beam splitting of light with the help of a birefringent prism. The splitting angle $\theta^{\prime}$ inside the prism is calculated in (11). The splitting angle $\theta$ of two beams after the prism is calculated in (15). Vectors $\boldsymbol{n}$ and $\boldsymbol{n}^{\prime}$ are normals to two interfaces, vector $\boldsymbol{l}$ is in the incidence plane and on the left interface, while $\boldsymbol{t}$ is orthogonal to the incidence plane. Vector $\boldsymbol{l}^{\prime}$ (not shown)is in the scattering plane and on the right interface.

We do not need to consider in details the boundary conditions to find reflection and refraction amplitudes, which depend on polarization of the incident beam. All that was considered in [6]. We need here only to find wave vectors of two modes, created in the anisotropic medium. There is no problem for $\boldsymbol{e}_{1}$ mode, since for it

$$
\boldsymbol{k}_{1}=\boldsymbol{l} k_{\|}+\boldsymbol{n} k_{1 \perp}, \quad k_{1 \perp}=\sqrt{\epsilon_{0} k_{0}^{2}-k_{\|}^{2}} .
$$

The problem is for $\boldsymbol{e}_{2}$ mode, for which $k_{2 \perp}$, defined as

$$
k_{2 \perp}=\sqrt{k_{0}^{2} \epsilon(\chi)-k_{\|}^{2}},
$$

does not determine the value of $k_{2 \perp}$, because it enters also the definition of the angle $\chi$, so to find the explicit dependence of $k_{2 \perp}$ on $\boldsymbol{a}$ it is necessary to solve the equation

$$
k_{\|}^{2}+x^{2}+\eta\left(k_{\|}(\boldsymbol{l} \cdot \boldsymbol{a})+x(\boldsymbol{n} \cdot \boldsymbol{a})\right)^{2}=k_{0}^{2} \epsilon_{0}(1+\eta),
$$


where $x$ denotes $k_{2 \perp}, \boldsymbol{n}$ is a unit vector of normal, directed toward isotropic medium, and $\boldsymbol{l}$ is a unit vector along $\boldsymbol{k}_{\|}$, which together with $\boldsymbol{n}$ constitutes the plane of incidence. Solution of this equation is

$$
x=\frac{-\eta k_{\|}(\boldsymbol{n} \cdot \boldsymbol{a})(\boldsymbol{l} \cdot \boldsymbol{a})+\sqrt{\epsilon_{0} k_{0}^{2}(1+\eta)\left(1+\eta(\boldsymbol{n} \cdot \boldsymbol{a})^{2}\right)-k_{\|}^{2}\left(1+\eta(\boldsymbol{l} \cdot \boldsymbol{a})^{2}+\eta(\boldsymbol{n} \cdot \boldsymbol{a})^{2}\right)}}{1+\eta(\boldsymbol{n} \cdot \boldsymbol{a})^{2}} .
$$

The sign chosen before square root provides the correct asymptotics at $\eta=0$ equal to isotropic value $\sqrt{\epsilon_{1} k_{0}^{2}-k_{\|}^{2}}$. In the following for simplicity we use the case, when $\boldsymbol{a}$ lies in the interface and directed at the angle $\pi / 2$ with respect to $\boldsymbol{l}$. Therefore $(\boldsymbol{n} \cdot \boldsymbol{a})=0$ and $(\boldsymbol{l} \cdot \boldsymbol{a})^{2}=0.5$, so we get

$$
k_{2 \perp}=\sqrt{\epsilon_{0} k_{0}^{2}(1+\eta)-k_{\|}^{2}(1+\eta / 2)} .
$$

In the case of calcite, where $\eta<0, k_{2} \perp<k_{1 \perp}$, the parallel to the interface components of the wave vectors inside and outside the prism are equal, but the normal components are

$$
k_{1 \perp}=\sqrt{\epsilon_{0} k_{0}^{2}-k_{\|}^{2}}, \quad k_{2 \perp}=\sqrt{\epsilon_{0} k_{0}^{2}(1+\eta)-k_{\|}^{2}\left(1+\eta(\boldsymbol{l} \cdot \boldsymbol{a})^{2}\right)} .
$$

The angular separation $\theta^{\prime}$ of two modes inside the crystal is found from

$$
\begin{gathered}
\cos \theta^{\prime}=\frac{k_{\|}^{2}+k_{1 \perp} k_{2 \perp}}{k_{1} k_{2}}=\frac{k_{\|}^{2}+\sqrt{\epsilon_{0} k_{0}^{2}-k_{\|}^{2}} \sqrt{\epsilon_{0} k_{0}^{2}(1+\eta)-k_{\|}^{2}(1+\eta / 2)}}{k_{0} \sqrt{\epsilon_{0}} \sqrt{\epsilon_{0} k_{0}^{2}(1+\eta)-k_{\|}^{2} \eta / 2}}= \\
=\frac{x+\sqrt{1-x} \sqrt{1-x+\eta(1-x / 2)}}{\sqrt{1+\eta(1-x / 2)}},
\end{gathered}
$$

where $x=\sin ^{2} \phi / \epsilon_{0}$ and $\phi$ is the incidence angle in vacuum. For the incident angle $\phi_{0}=0.6$ we can find $\theta^{\prime} \approx 0.1$.

At the exit through the right facet with vectors $\boldsymbol{n}^{\prime}=\boldsymbol{n} \cos \psi+\boldsymbol{l} \sin \psi, \boldsymbol{l}^{\prime}=\boldsymbol{l} \cos \psi-\boldsymbol{n} \sin \psi$ in transmission plane the parallel to the exit interface components of two modes wave vectors are $k_{1 \|}=$ $\left(k_{\|} \boldsymbol{l}+k_{1 \perp} \boldsymbol{n}\right) \cdot \boldsymbol{l}^{\prime}=k_{\|} \cos \psi-k_{1 \perp} \sin \psi, k_{2 \|}=\left(k_{\|} \boldsymbol{l}+k_{2 \perp} \boldsymbol{n}\right) \cdot \boldsymbol{l}^{\prime}=k_{\|} \cos \psi-k_{2 \perp} \sin \psi, k_{01 \perp}=\sqrt{k_{0}^{2}-k_{1 \|}^{2}}$, $k_{02 \perp}=\sqrt{k_{0}^{2}-k_{2 \|}^{2}}$. Therefore the angular divergence of two beams after exit from the prism is

$$
\cos \theta=\left[k_{01 \perp} k_{02 \perp}+k_{1 \|} k_{2 \|}\right] / k_{0}=\left[\sqrt{k_{0}^{2}-k_{1 \|}^{2}} \sqrt{k_{0}^{2}-k_{2 \|}^{2}}+k_{1 \|} k_{2 \|}\right] / k_{0}^{2}
$$

where

$$
\begin{gathered}
k_{1 \|}=k_{\|} \cos \psi-\sqrt{\epsilon_{0} k_{0}^{2}-k_{\|}^{2}} \sin \psi=k_{0}\left[x \cos \psi-\sin \psi \sqrt{\epsilon_{0}-x^{2}}\right], \quad x=\sin \phi \\
k_{2 \|}=k_{\|} \cos \psi-\sqrt{\epsilon_{0} k_{0}^{2}(1+\eta)-k_{\|}^{2}\left(1+\eta(\boldsymbol{l} \cdot \boldsymbol{a})^{2}\right)} \sin \psi= \\
=k_{0}\left[x \cos \psi-\sin \psi \sqrt{\epsilon_{0}(1+\eta)-x^{2}(1+\eta / 2)}\right]
\end{gathered}
$$

Therefore

$$
\begin{gathered}
\cos \theta=\left[k_{01 \perp} k_{02 \perp}+k_{1 \|} k_{2 \|}\right] / k_{0}^{2}= \\
=\left[\sqrt{1-\left[x \cos \psi-\sin \psi \sqrt{\epsilon_{0}-x^{2}}\right]^{2}} \sqrt{1-\left[x \cos \psi-\sin \psi \sqrt{\epsilon_{0}(1+\eta)-x^{2}(1+\eta / 2)}\right]^{2}}+\right. \\
\left.+\left[x \cos \psi-\sin \psi \sqrt{\epsilon_{0}-x^{2}}\right]\left[x \cos \psi-\sin \psi \sqrt{\epsilon_{0}(1+\eta)-x^{2}(1+\eta / 2)}\right]\right] .
\end{gathered}
$$

Dependence of the divergence angle $\theta(\phi)$ is shown in Fig.3. 


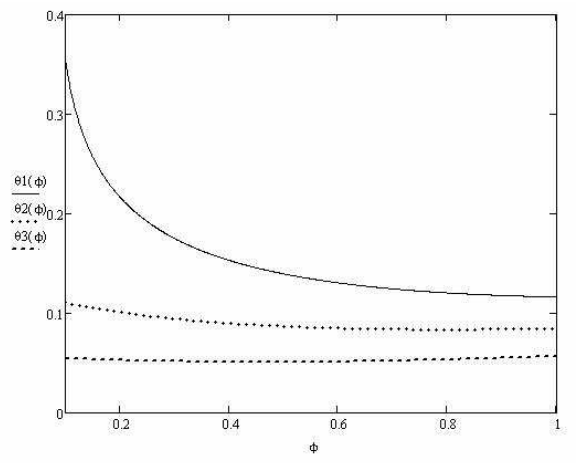

Figure 3. Beam splitting of light exiting from the birefringent prism shown in Fig.2 in dependence on incident angle $\phi$ and the angle $\psi$ between normals $\boldsymbol{n}$ and $\boldsymbol{n}^{\prime}$. The curves $\theta 1,2,3(\phi)$ correspond to $\psi=0.7 ; 0.5 ; 0.3$ respectively.

\section{Discussion}

We have calculated here the angular divergence of beams after transmission through a linear birefringent calcite crystal. There are no down conversion. Therefore two beams represent a single photon and one can easily observe an interference after superposition of the beams in one of the detectors. Nevertheless the photons are Bose particles. Therefore one cannot take for granted that the two beams correspond to a single photon. It can be that the two beams are related to two photons and these photons are entangled. Therefore one can expect that coincidence measurements will show nonzero result. It will depend on intensity of the incident beam. It is also interesting to look what result will be found in measurement of the Bell inequality in this scheme. The advantage of this scheme is the possibility of variation of the relative two beams intensity by varying polarization of the incident beam. And everything can be calculated analytically [6].

\section{References}

1. J. Brendel, E. Mohler, and W. Martienssen "Time-Resolved Dual-Beam Two-Photon Interferences with High Visibility" Phys.Rev.Lett. 66 1142-47 (1991).

2. David C. Burnham and Donald L. Weinberg "OBSERVATION OF SIMULTANEITY IN PARAMETRIC PRODUCTION OF OPTICAL PHOTON PAIRS" Phys.Rev.Lett. $2584-87$ (1970).

3. C. K. Hong, Z. Y. au, and L. Mandel "Measurement of Subpicosecond Time Intervals between Two Photons by Interference" Phys.Rev.Lett. 59 2044-46 (1987).

4. Z. Y. Ou and L. Mandel "Violation of Bell's Inequality and Classical Probability in a Two-Photon Correlation Experiment" Phys.Rev.Lett. 61 50-53 (1988).

5. R. Hanbury Brown and R. Q. Twiss "Interferometry of the intensity fluctuations in light. I. Basic theory: the correlation between photons in coherent beams of radiation". Proc of the Royal Society of London bf A 242 300-324 (1957).

6. F V Ignatovich, V K Ignatovich. "Bulk and surface plane electromagnetic waves in anisotropic media" https://arxiv.org/pdf/1101.0829v4.pdf; "Optics of anisotropic media." Physics-Uspekhi. v.55, pp.709-720 $(2012)$ 